\title{
Bone Marrow Edema and Joint Effusion Evaluation in Various Stages of Avascular Necrosis of Femoral Head using Magnetic Resonance Imaging
}

\author{
K. S. Vedaraju ${ }^{1}$, Supreetha Hegde $A .^{2}$ \\ ${ }^{1}$ Professor, Department of Radiology, ${ }^{2}$ Resident, Department of Radiology, Bangalore Medical College and Research Institute, \\ Bangalore, Karnataka, India
}

Corresponding author: Dr. Supreetha Hegde A., "Suneetha", near bus stop, Kuntalpady junction, Karkala-574104, Udupi district, Karnataka, India.

DOI: http://dx.doi.org/10.21276/ijcmsr.2019.4.1.11

How to cite this article: K. S. Vedaraju, Supreetha Hegde A. Bone marrow edema and joint effusion evaluation in various stages of avascular necrosis of femoral head using magnetic resonance imaging. International Journal of Contemporary Medicine Surgery and Radiology. 2019;4(1):A42-A46.

\section{A B S T R A C T}

Introduction: Bone marrow edema and joint effusion are common occurrences in femoral head avascular necrosis. MRI is an invaluable tool in their evaluation. The objective of this study was to determine the occurrence of bone marrow edema and joint effusion and their relationship to stage of femoral head avascular necrosis using Magnetic Resonance Imaging.

Material and Methods: 55 patients having avascular necrosis of femoral head based on characteristic radiographic and Magnetic Resonance Imaging findings were studied. Informed consent was taken from all patients. A total of 110 hips were studied, staged using Ficat-Arlet system and evaluated for bone marrow edema and joint effusion using Magnetic Resonance Imaging. Bone marrow edema is defined as an ill-defined low signal intensity area on T1-weighted images with corresponding high signal intensity on T2-weighted/inversion recovery images. Grading of hip joint effusion was done from 0 to 3 based on the amount of fluid present.

Results: Out of 55 patients, 25 patients had bilateral disease. Bone marrow edema was present in 44 out of 110 hips studied and occurred most often in stage III disease. Joint effusion was present in 46 out of 110 hips studied. Significant joint effusion (>/= grade II) showed maximum occurrence in stage III disease. All patients with grade III joint effusion had stage III disease. Conclusions: Peak occurrence of bone marrow edema and joint effusion occurs in Ficat-Arlet stage III avascular necrosis. Significant joint effusion occurs in later stages of the disease with maximum occurrence and severe grade in stage III.

Keywords: Ficat-Arlet System, Radiographic, Signal Intensity, Significant Joint Effusion, T2-Weighted/Inversion Recovery

\section{INTRODUCTION}

\section{Background}

Avascular necrosis (AVN) of femoral head is an increasingly common cause of musculoskeletal disability, posing a major diagnostic and therapeutic challenge. ${ }^{1}$ Magnetic resonance imaging is the most sensitive means of diagnosing avascular necrosis. ${ }^{2}$ There are various staging systems for classification of avascular necrosis. The staging system developed by Ficat and Arlet and has been used widely for treating avascular necrosis. ${ }^{3}$ Bone marrow edema detected on magnetic resonance imaging has been considered as a marker of progression to collapse in avascular necrosis of the femoral head. ${ }^{4}$ The amount of joint effusion, with distension of the capsular recess in grade III effusion, by has been well correlated with the radiographic stages of AVN of the femoral head. ${ }^{5}$ Hence detection of bone marrow edema and joint effusion in relation to various stages of femoral head avascular necrosis will have significant therapeutic and prognostic implications.

Study objective was to determine the occurrence of bone marrow edema and joint effusion and their relationship to various stages of avascular necrosis of femoral head using Magnetic Resonance Imaging.

\section{MATERIAL AND METHODS}

A cross-sectional study was done from November 2016 to November 2018.55 patients, aged above 18 years, presenting to Victoria Hospital attached to Bangalore Medical College and Research Institute, Bangalore having non-traumatic avascular necrosis of femoral head based on characteristic radiographic and magnetic resonance imaging findings were included in the study. Patients presenting with traumatic pathology and those with contraindications to magnetic resonance imaging were excluded. Ethical committee clearance was obtained. Informed consent was taken from all patients. There were 43 males and 12 females in the study. A total of 110 hips were evaluated. 25 patients had bilateral hip involvement and 30 patients had unilateral hip involvement.

\section{Imaging techniques}

All magnetic resonance imaging examinations were 
performed on SIEMENS Magnetom Avanto 1.5-T magnet system. Imaging was performed using a body coil placed over the pelvis with the patient in supine position and both hips were examined simultaneously. The following sequences were obtained: coronal spin-echo T1-weighted (TR range/ TE range, 450-650/16-20), intermediate-weighted (1500-2200/20-40), T2-weighted (1500-2200/ 80-90), and inversion recovery (2500-3000/35-40; inversion time, 100-150) sequences of both hips. Subsequent intermediateweighted and T2-weighted images were obtained in the sagittal and axial planes using the same parameters as those in the coronal plane. Axial gradient echo imaging was performed when required. Gadolinium enhanced fat supressed T1 weighted images were obtained in few selected patients with suspected stage I disease. Slice thickness was 1-5 $\mathrm{mm}$, with an inter-slice gap of $0.5 \mathrm{~mm}$.

\section{Image interpretation}

The diagnosis of femoral head avascular necrosis was established on the basis of characteristic radiographic and magnetic resonance imaging findings with staging done according to Ficat-Arlet system. The patients underwent antero-posterior radiograph and magnetic resonance imaging of bilateral hips.

The following were the magnetic resonance imaging criteria used to diagnose femoral head avascular necrosis: ${ }^{6}$

- Serpigenious line/ crescent shaped area of low signal intensity in the weight-bearing portion of the femoral head (Figure 1)

- A band-like pattern in the bone marrow corresponding to sclerotic change on radiograph

- Double line sign: Inner bright T2 line representing granulation tissue and adjacent dark $\mathrm{T} 2$ line representing sclerotic bone. (Figure 2)

- A ring-like lesion with homogeneous or heterogeneous signal intensity of the central area

- A homogeneous area of decreased signal intensity with a dark band

- Collapse of the femoral head (Figure 2)

\section{Criteria to diagnose bone marrow edema}

Bone marrow edema was defined as an ill-defined area of low signal intensity on T1-weighted images with corresponding high signal intensity on T2-weighted or inversion recovery images. ${ }^{6-11}$ (Figure 1) It was localised to the head, neck and/ or intertrochanteric region of femur. Bone marrow edema was not seen in the acetabulum.

A well-demarcated arcuate zone of high signal intensity on T2-weighted images surrounding the necrotic area of the femoral head was considered granulation tissue. , $^{6,11 .}$

\section{Grading of joint effusion}

The joint fluid was graded from 0 to 3 on the basis of the coronal STIR or T2W images as follows: Grade 0, no fluid in joint space; Grade 1, minimal joint effusion - minimal fluid in joint space (Figure 1); Grade 2, moderate joint effusion - enough fluid to surround the femoral neck; and Grade 3 , severe joint effusion -fluid causing distention of capsule recesses $^{6}$ (Figure 1).

\section{Staging of $\mathrm{AVN}^{3}$}

The staging system proposed by Ficat-Arlet was used.
Stage 0: Preclinical: Normal plain radiograph, Normal MRI Stage I: Pre-radiographic: Normal X-ray, Abnormal MRIodema, Bone scan: Increased uptake

Stage II: Pre-collapse (reparative stage before flattening occurs) Diffuse sclerosis of femoral head on X ray. Geographic defect in femoral head on MRI 'Double line sign'

Stage III: Collapse: Subchondral fracture- 'crescent sign' on $\mathrm{X}$ ray, Eventual cortical collapse on MRI

Stage IV: Osteoarthritis: Femoral head collapse with secondary degenerative changes, Acetabular involvement.

A total of 110 hips were studied, staged using FicatArlet system and evaluated for bone marrow edema and joint effusion using Magnetic Resonance Imaging. Image interpretation was done by two experienced radiologists.

\section{STATISTICAL ANALYSIS}

Logistic regression was used to assess the association between the occurrence of bone marrow edema and the stage of AVN. This included Pearson's correlation coefficient and chi-square tests. A $P$ value of less than 0.05 indicated a statistically significant difference.

Microsoft Exel and Epi info were used for data analysis. Results on continuous measurements are presented on Mean $\pm \mathrm{SD}$ (Min-Max) and results on categorical measurements are presented in Number (\%).

\section{RESULTS}

Out of 55 patients, 43 were males and 12 were females. The mean age of presentation was $38.53+/-13.16$ (21-69). Bilateral disease was present in 25 patients. Unilateral disease was present in 30 patients. Out of 110 hips of 55 patients evaluated, 80 hips were affected by AVN and 30 hips were unaffected (stage 0 ). Male preponderance of the disease was noted.

Bone marrow edema was present in 44 out of 110 hips studied. It was seen most often in stage III disease $(18 / 25,40.9 \%)$, compared to stage I $(1 / 3,2.3 \%)$, stage II $(13 / 26,29.5 \%)$ and

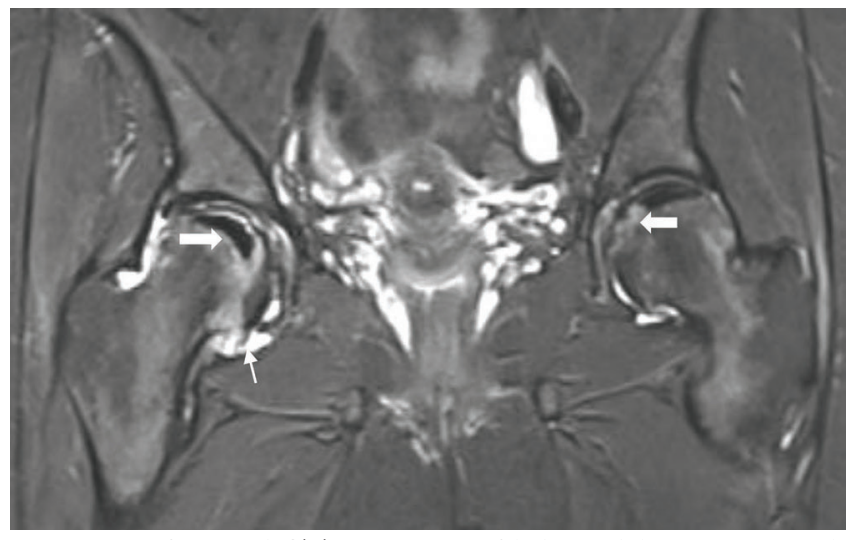

Figure-1: Coronal STIR image of bilateral hip joints with AVN Ficat-Arlet stage III on both sides in a 30-year-old female. Crescent area (solid arrow) of low signal intensity at weight-bearing portion of bilateral femoral head. Bone marrow edema seen in the right femoral head, left femoral head, neck and lesser trochanteric region. Grade 3 joint effusion (arrow) that distended capsular recess in the right side and grade 1 joint effusion in the left side. 


\begin{tabular}{|c|c|c|c|c|c|}
\hline \multicolumn{6}{|l|}{ Cross-table } \\
\hline & & & \multicolumn{2}{|c|}{ BME } & \multirow[t]{2}{*}{ Total } \\
\hline & & & Absent & Present & \\
\hline \multirow[t]{10}{*}{ AVN } & \multirow[t]{2}{*}{ Stage 0} & Count & 30 & 0 & 30 \\
\hline & & $\%$ & $45.5 \%$ & $0.0 \%$ & $27.3 \%$ \\
\hline & \multirow[t]{2}{*}{ Stage I } & Count & 2 & 1 & 3 \\
\hline & & $\%$ & $3.0 \%$ & $2.3 \%$ & $2.7 \%$ \\
\hline & \multirow[t]{2}{*}{ Stage II } & Count & 13 & 13 & 26 \\
\hline & & $\%$ & $19.7 \%$ & $29.5 \%$ & $23.6 \%$ \\
\hline & \multirow[t]{2}{*}{ Stage III } & Count & 7 & 18 & 25 \\
\hline & & $\%$ & $10.6 \%$ & $40.9 \%$ & $22.7 \%$ \\
\hline & \multirow[t]{2}{*}{ Stage IV } & Count & 14 & 12 & 26 \\
\hline & & $\%$ & $21.2 \%$ & $27.3 \%$ & $23.6 \%$ \\
\hline \multirow{2}{*}{\multicolumn{2}{|c|}{ Total }} & Count & 66 & 44 & 110 \\
\hline & & $\%$ & $100.0 \%$ & $100.0 \%$ & $100.0 \%$ \\
\hline \multicolumn{6}{|l|}{ Chi-Square Test } \\
\hline & \multicolumn{2}{|c|}{ Value } & $\mathrm{df}$ & \multicolumn{2}{|c|}{$P$ value (significant if $<0.05$ ) } \\
\hline Pearson Chi-Square & \multicolumn{2}{|c|}{32.216} & 4 & \multicolumn{2}{|c|}{$<0.001$} \\
\hline $\mathrm{N}$ of Valid Cases & \multicolumn{2}{|c|}{110} & & \multicolumn{2}{|c|}{ Avascular necrosis (AVN) of femor } \\
\hline
\end{tabular}

\begin{tabular}{|c|c|c|c|c|c|c|c|c|}
\hline \multicolumn{9}{|c|}{ Cross-table } \\
\hline & & & \multicolumn{4}{|c|}{ JE } & \multirow[t]{2}{*}{ Total } & \multirow{2}{*}{$\begin{array}{c}\text { Sig. JE } \\
(=/> \\
\text { grade II) }\end{array}$} \\
\hline & & & Grade 0 & Grade I & Grade II & Grade III & & \\
\hline \multirow[t]{10}{*}{ AVN stage } & \multirow[t]{2}{*}{0} & Count & 30 & 0 & 0 & 0 & 30 & 0 \\
\hline & & $\%$ & $46.9 \%$ & $0.0 \%$ & $0.0 \%$ & $0.0 \%$ & $27.3 \%$ & \\
\hline & \multirow[t]{2}{*}{1} & Count & 3 & 0 & 0 & 0 & 3 & 0 \\
\hline & & $\%$ & $4.7 \%$ & $0.0 \%$ & $0.0 \%$ & $0.0 \%$ & $2.7 \%$ & \\
\hline & \multirow[t]{2}{*}{ II } & Count & 14 & 12 & 0 & 0 & 26 & 0 \\
\hline & & $\%$ & $21.9 \%$ & $41.4 \%$ & $0.0 \%$ & $0.0 \%$ & $23.6 \%$ & \\
\hline & \multirow[t]{2}{*}{ III } & Count & 3 & 7 & 10 & 5 & 25 & 15 \\
\hline & & $\%$ & $4.7 \%$ & $24.1 \%$ & $83.3 \%$ & $100.0 \%$ & $22.7 \%$ & \\
\hline & \multirow[t]{2}{*}{ IV } & Count & 14 & 10 & 2 & 0 & 26 & 2 \\
\hline & & $\%$ & $21.9 \%$ & $34.5 \%$ & $16.7 \%$ & $0.0 \%$ & $23.6 \%$ & \\
\hline \multirow{2}{*}{\multicolumn{2}{|c|}{ Total }} & Count & 64 & 29 & 12 & 5 & 110 & 17 \\
\hline & & $\%$ & $100.0 \%$ & $100.0 \%$ & $100.0 \%$ & $100.0 \%$ & $100.0 \%$ & \\
\hline \multicolumn{9}{|c|}{ Chi-Square Tests } \\
\hline & & \multicolumn{2}{|c|}{ Value } & & \multicolumn{2}{|l|}{ df } & \multicolumn{2}{|c|}{$P$ value (significant if $<0.05$ ) } \\
\hline \multicolumn{2}{|c|}{ Pearson Chi-Square } & \multicolumn{2}{|c|}{76.359} & & \multicolumn{2}{|l|}{12} & \multicolumn{2}{|c|}{$<0.001$} \\
\hline \multicolumn{2}{|c|}{$\mathrm{N}$ of Valid Cases } & \multicolumn{2}{|c|}{110} & & & & & \\
\hline
\end{tabular}

stage IV (12/26, 27.3\%). Bone marrow edema was noted in femoral head, neck and/or intertrochanteric regions. No case showed bone marrow edema in the acetabulum. Occurrence of bone marrow edema in various stages of femoral head avascular necrosis showed an increasing trend from stage I to stage III, with peak occurrence in stage III; and showed decrease in stage IV disease where there is development of significant femoral head collapse and degenerative changes in the joint.

There was a strong association between the presence of bone marrow edema and significant joint effusion ( $\operatorname{grade}=/>2$ ), independent of stage of avascular necrosis of the femoral head $(p<0.001)$. The odds ratio for bone marrow edema with respect to significant joint effusion was 16.55. Among 44 hips with bone marrow edema, 15 hips (34\%) had significant joint effusion.

Joint effusion was present in 46 out of 110 hips studied. The later stages (III, IV) of

femoral head avascular necrosis showed presence of significant joint effusion (>/= grade II). Out of 17 hips with significant joint effusion, 15 hips had stage III disease and 2 hips has stage IV disease. Hence, significant joint effusion showed maximum occurrence in stage III disease $(15 / 25$, $60 \%)$, compared to stage I $(0 / 3,0 \%)$, stage II $(0 / 26,0 \%)$ and stage IV (2/26, 7.69\%). All patients with grade 3 joint effusion (5 hips) had stage III disease. 


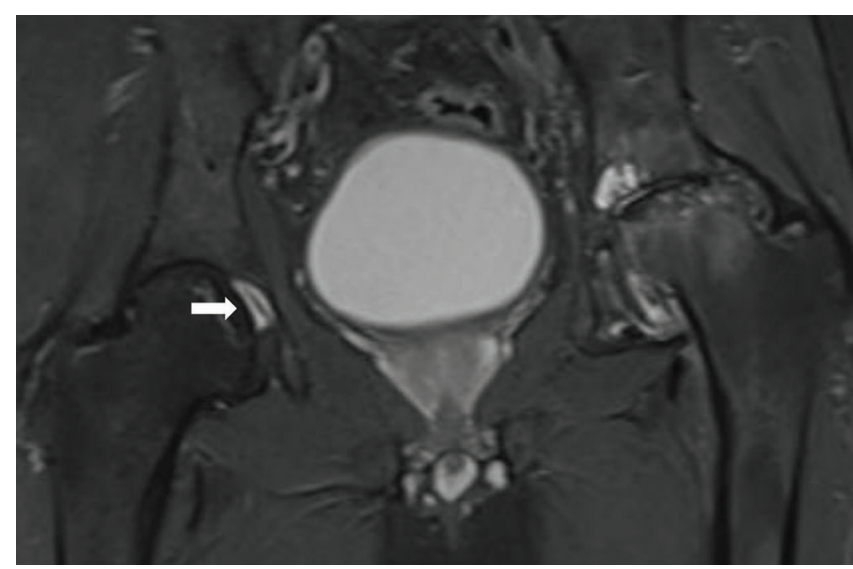

Figure-2: Coronal STIR image of bilateral hip joints with AVN Ficat-Arlet stage II on right side and stage IV on left side in a 50-year-old male. Double line sign (solid arrow) in right femoral head with no bone marrow edema and no joint effusion. Collapse of left femoral head with subchondral cysts and marginal osteophytes.

29 hips had a grade I joint effusion with $12 / 26$ in stage II, $7 / 25$ in stage III and 10/26 in stage IV disease.

\section{DISCUSSION}

Bone marrow edema can occur in several conditions like femoral head avascular necrosis, Transient osteoporosis of the hip, transient bone marrow syndrome, bone bruise etc. Bone marrow edema can occur due to subchondral fracture, mechanical stress or intramedullary venous stasis. ${ }^{6}$ In 2000, Kim et al., in a study titled "The pattern of bone marrow oedema on MRI in osteonecrosis of the femoral head" on 200 patients with non-traumatic AVN concluded that the initial abnormal finding detected on MRI was a band of abnormal intensity at the junction between the necrotic and the normal area. ${ }^{8}$ They also observed that the bone marrow edema pattern was not observed in early stages of the disease and that the structural damage seemed to bring about the marrow oedema pattern and pain. In 2008, Ragab Y. et al., in a study titled "Bone marrow edema syndromes of the hip: MRI features in different hip disorders "on 34 patients, evaluated the role of MRI in the evaluation of bone marrow edema syndromes affecting the hip joint in patients with hip pain. ${ }^{12}$ They found that in around $30 \%$ of the patients, avascular necrosis was the cause of hip pain. They showed that MRI is the modality of choice when hip disease is suspected clinically and plain radiographs are normal or equivocal. Some investigators have shown that patients who present with diffuse bone marrow edema in the femoral neck and intertrochanteric region may subsequently develop focal lesions in the femoral head consistent with osteonecrosis. ${ }^{13}$ However, in our study, all hips exhibited focal subchondral lesions characteristic of femoral head avascular necrosis independent of whether or not it was associated with bone marrow edema.

In our study, bone marrow edema was present in $40 \%$ hips evaluated for avascular necrosis of femoral head. Our figure is similar to those previously reported by other investigators, which have ranged from $30 \%$ to $50 \% .^{7,8,9} \mathrm{Kim}$ et al. ${ }^{8}$ reported that bone marrow edema was most often found in hips with stage III disease (88\%). Iida et al. ${ }^{9}$ observed that bone marrow edema correlated highly with hips that progressed to collapse of the femoral head. Our results corroborate that the peak occurrence of bone marrow edema is in stage III disease (40.9\%), when compared to other stages. According to some investigators bone marrow edema could be regarded as a reaction to subchondral fractures which arise from mechanical stress. ${ }^{6,7,9}$ Medullary space histology in the affected hips has shown eosinophilic plasma-like fluid around the necrotic fat cells attributing to bone marrow edema. ${ }^{6,7}$ In few cases, bone marrow edema showed correlation to fibrotic areas, dilated blood vessels and interstitial edema around the adipocytes ${ }^{6,7,11}$ Ficat and Arlet postulated that intramedullary stasis in femoral head and intertrochanteric region secondary to the venous drainage block resulted in bone marrow edema. ${ }^{14}$

Our data showed an increasing trend of occurrence of bone marrow edema in femoral head avascular necrosis from stage I to stage III. Peak occurrence occurred in stage III which is consistent with the findings of previous investigators. With the development of significant femoral head collapse and degenerative changes in the joint in stage IV, bone marrow edema showed a decreasing trend.

A grade 2 or grade 3 effusion occurred in 17 of hips studied. Our data indicates that the largest prevalence of significant joint effusion occurred in hips with stage III disease. This finding is similar to that of a prior study by Mitchell et al. ${ }^{10}$, which reported that $58 \%$ of hips with osteonecrosis of the femoral head had grade 2 or grade 3 effusions. Our results also indicated that the occurrence of bone marrow edema was 16.55 times in odds ratio more likely to occur when there was a joint effusion in the hip affected by osteonecrosis of the femoral head. Grade 3 joint effusion in our study was seen only in patients with stage III disease.

There were several limitations to our study. A detailed clinical correlation was not carried out with the imaging parameters assessed. The cause of pain in patients with osteonecrosis of the femoral head is not well understood. A prospective study of patients with a logged pain scale and a series of magnetic resonance images would be helpful to determine the relationship of bone marrow edema and effusions to hip pain in patients with osteonecrosis of the femoral head. It is difficult to obtain patients with bone marrow edema and joint effusions

but without osteonecrosis of the femoral head as control subjects for the study. Uninvolved contralateral hip in the study group will constitutes suboptimal control group.

In summary, bone marrow edema which is enhanced in the presence of joint effusion, and joint effusion alone in femoral head avascular necrosis, are most likely to occur in stage III disease and with increased severity showing strong statistical correlation.

In 2003, Guo-Shu Huang et al, in a study titled "MR Imaging of Bone Marrow Edema and Joint Effusion in Patients with Osteonecrosis of the Femoral Head: Relationship to Pain" studied the occurrence of bone marrow edema and joint effusion and their relationship to the stage of AVN and pain. ${ }^{6}$ They concluded that both bone marrow edema and joint effusions existed with a peak occurrence in stage III 
disease and both were most likely to occur when the patient presented with pain. Our data supports these observations. Studies have suggested that occurrence of bone marrow edema and joint effusion in avascular necrosis of femoral head may suggest impending progression to subchondral cortical collapse. Hence disease management needs detailed analysis of imaging data and staging, with stage III disease being important for therapeutic considerations. Our study also corroborates with the data depicting significant association of bone marrow edema and joint effusion in stage III of avascular necrosis of femoral head, with magnetic resonance imaging being the most invaluable tool in the evaluation of the same.

Though bone marrow edema and joint effusion are nonspecific findings seen in femoral head avascular necrosis, our study has re-emphasised the importance of evaluation of these features on magnetic resonance imaging and has consistently arrived at statistically significant associations with staging of the disease.

\section{CONCLUSION}

Peak occurrence of bone marrow edema and joint effusion occurs in Ficat-Arlet stage III avascular necrosis. Significant joint effusion occurs in later stages of the disease with maximum occurrence and severe grade in stage III. The clinical and therapeutic implications of these features can have prospects in contributing to more efficient disease management and improved prognosis.

\section{REFERENCES}

1. Yochum T, Rowe L. Essentials of skeletal radiology. 3rd ed. Lippincot: Williams \& Wilkins 2005; 114-115.

2. Mitchell M, Kundel H, Steinberg M, Kressel H, Alavi A, Axel L. Avascular necrosis of the hip: comparison of MR, CT, and scintigraphy. American Journal of Roentgenology. 1986;147(1):67-71.

3. Khanna A, Yoon T, Mont M, Hungerford D, Bluemke D. Femoral Head Osteonecrosis: Detection and Grading by Using a Rapid MR Imaging Protocol. Radiology. 2000;217(1):188-192.

4. Iida S, Harada Y, Shimizu K, et al. Correlation between bone marrow edema and collapse of the femoral head in steroid-induced osteonecrosis. American Journal of Roentgenology. 2000; 174(3):735-743

5. Mitchell DG, Rao V, Dalinka M, et al. MRI of joint fluid in the normal and ischemic hip. American Journal of Roentgenology. 1986; 146(5):1215-1218

6. Huang G, Chan W, Chang Y, Chang C, Chen C, Yu J. MR Imaging of Bone Marrow Edema and Joint Effusion in Patients with Osteonecrosis of the Femoral Head: Relationship to Pain. American Journal of Roentgenology. 2003;181(2):545-549.

7. Koo KH, Ahn IO, Kim R, et al. Bone marrow edema and associated pain in early stage osteonecrosis of the femoral head: prospective study with serial MR images. Radiology 1999; 213(1):715-722

8. Kim $\mathrm{Y}, \mathrm{Oh} \mathrm{H,} \mathrm{Kim} \mathrm{H.} \mathrm{The} \mathrm{pattern} \mathrm{of} \mathrm{bone} \mathrm{marrow}$ oedema on MRI in osteonecrosis of the femoral head. The Journal of Bone and Joint Surgery British volume. 2000;82-B (6):837-841.
9. Iida S, Harada Y, Shimizu K, et al. Correlation between bone marrow edema and collapse of the femoral head in steroid-induced osteonecrosis. American Journal of Roentgenology. 2000; 174(5):735-743

10. Mitchell DG, Steinberg ME, Dalinka MK, Rao VM, Fallon M, Kressel HY. Magnetic resonance imaging of the ischemic hip: alterations within the osteonecrosis, viable, and reactive zones. Clinical Orthopaedics 1989; 244(2):60-77

11. Sakai T, Sugano N, Nishii T, Haraguchi K, Ochi T, Ohzono K. MR findings of necrotic lesions and the extralesional area of osteonecrosis of the femoral head. Skeletal Radiology 2000; 29(4):133-141

12. Ragab Y, Emad Y, Abou-Zeid A. Bone marrow edema syndromes of the hip: MRI features in different hip disorders. Clinical Rheumatoly. 2008;27(4):475-82.

13. Turner DA, Templeton AC, Selzer PM, Rosenberg AG, Petasnick JP. Femoral capital osteonecrosis: MR finding of diffuse marrow abnormalities without focal lesions. Radiology 1989; 171(6):135-140

14. Ficat RP, Arlet J. Necrosis of the femoral head. In: Hungerford DS, ed. Ischemia and necrosis of bone. Baltimore: Williams \& Wilkins, 1980:11-52

Source of Support: Nil; Conflict of Interest: None

Submitted: 01-02-2019; Accepted: 02-03-2019; Published online: 13-03-2019 